\title{
Myeloid Cells to the Rescue: Improving Thrombus Resolution
}

\author{
Tanja Knopp ${ }^{1}$ Susanne Karbach ${ }^{1,2,3}$ Philip Wenzel ${ }^{1,2,3}$
}

${ }^{1}$ Center for Thrombosis and Hemostasis, University Medical Center Mainz, Mainz, Germany

${ }^{2}$ Center for Cardiology-Cardiology 1, University Medical Center Mainz, Mainz, Germany

${ }^{3}$ Deutsches Zentrum für Herz- und Kreislaufforschung e.V. (DZHK) Partner Site Rhine-Main, University Medical Center Mainz, Mainz, Germany

Thromb Haemost 2020;120:197-198.

Venous thromboembolism (VTE) comprising deep vein thrombosis and pulmonary embolism is one of the leading causes of morbidity and mortality in the world. ${ }^{1}$ Therapy today means anticoagulation, which also puts the patient at significant bleeding risk. The number of patients afflicted by VTE indicates that pathophysiology of this disease is not yet fully understood and must be further investigated to provide better strategies for prevention and treatment and to avoid recurrence. In this issue, Kimball et al contribute to a better understanding of the role of monocytes/macrophages (Mo/ $\mathrm{M} \Phi$ ) in thrombus formation and resolution. ${ }^{2}$ They were able to show that toxin-mediated depletion of 80 to $90 \%$ of the $\mathrm{CD} 11 \mathrm{~b}^{+} \mathrm{Mo} / \mathrm{M} \Phi$ had no effects on neutrophil recruitment, formation of neutrophil extracellular traps, and venous thrombus formation in a VTE mouse model of vena cava ligation. While thrombogenesis was unaltered, depletion of circulating $\mathrm{CD} 11 \mathrm{~b}^{+} \mathrm{Mo} / \mathrm{M} \Phi$ resulted in larger thrombi and impaired intrathrombotic fibrinolysis at day 8 post ligation. This phenomenon seemed to be based on removing one of the main cellular components of a dissolving thrombus, the "reparative" Ly6C $\mathrm{C}^{\mathrm{lo}}$ monocytes. To test their hypothesis, $\mathrm{CD} 11 \mathrm{~b}^{+}$Ly6 $\mathrm{C}^{\text {lo }}$ monocytes were adoptively transferred into $\mathrm{Mo} / \mathrm{M} \Phi$ depleted mice, resulting in thrombus size comparable to nondepleted controls. In their model, the authors did not provide us with longitudinal data on thrombus resolution over time. Nevertheless, they corroborate earlier reports which showed that skewing the monocyte phenotype away from a Ly6C $\mathrm{C}^{\text {hi }} / \mathrm{IL}-12^{+} / \mathrm{T}_{\text {-bet }}{ }^{+}$subset with ensuing reduction of interferon gamma formation improves thrombus resolution. $^{3-5}$ Individuals suffering from inflammatory bowel disease or active autoimmune disease, in particular when complicated by antiphospholipid antibody syndrome, are at risk to experience VTE recurrence. ${ }^{1}$ Rosuvastatin admin-

Address for correspondence Philip Wenzel, MD, CTH Professorship Vascular Inflammation, and Center for Cardiology, Cardiology I, University Medical Center Mainz, Langenbeckstr. 1, 55131 Mainz, Germany (e-mail: wenzelp@uni-mainz.de).

istered to individuals with increased high-sensitive C-reactive protein reduced first occurrence of symptomatic VTE, ${ }^{6}$ suggesting that modification of the inflammatory burden might represent a valid drug therapy to prevent thrombosis. Further research will be needed to affirm that immunemodulatory strategies based on enhancing the reparative functions of myeloid cells will not only improve healing but also prevent recurrence of VTE.

\section{Funding}

This work was supported by the German Federal Ministry for Education and Research (BMBF 01E01503) and the Boehringer-Ingelheim Foundation.

\section{Conflict of Interest}

P.W. reports personal fees from Bayer, Astra-Zeneca, from Abbott, and Daiichi-Sankyo, personal fees and nonfinancial support from Novartis and Servier, outside the submitted work. All the other authors report no conflict of interest.

\section{References}

1 Konstantinides SV, Meyer G, Becattini C, et al; ESC Scientific Document Group. 2019 ESC Guidelines for the diagnosis and management of acute pulmonary embolism developed in collaboration with the European Respiratory Society (ERS). Eur Heart J 2019. Doi: 10.1093/eurheartj/ehz405

2 Kimball AS, Obi AT, Luke CE, et al. Ly6CLo Monocyte/Macrophages are Essential for Thrombus Resolution in a Murine Model of Venous Thrombosis. Thromb Haemost 2020;120(02): 289-299

3 Nosaka M, Ishida Y, Kimura A, et al. Absence of IFN- $\gamma$ accelerates thrombus resolution through enhanced MMP-9 and VEGF expression in mice. J Clin Invest 2011;121(07):2911-2920

(c) 2020 Georg Thieme Verlag KG Stuttgart · New York
DOI https://doi.org/ $10.1055 / \mathrm{s}-0039-1701044$. ISSN 0340-6245. 
4 Schönfelder T, Brandt M, Kossmann S, et al. Lack of T-bet reduces monocytic interleukin-12 formation and accelerates thrombus resolution in deep vein thrombosis. Sci Rep 2018;8 (01):3013

5 Luther N, Shahneh F, Brähler M, et al. Innate effector-memory $\mathrm{t}$-cell activation regulates post-thrombotic vein wall inflam- mation and thrombus resolution. Circ Res 2016;119(12): 1286-1295

6 Glynn RJ, Danielson E, Fonseca FA, et al. A randomized trial of rosuvastatin in the prevention of venous thromboembolism. $\mathrm{N}$ Engl J Med 2009;360(18):1851-1861 